\title{
Clinical Characteristics, Diagnosis and Management of Neonatal
}

\section{Stroke}

\author{
Jing Liu ${ }^{1,{ }^{*}}$, Ru-Xin Qiu ${ }^{1,2}$, Jian-Jun Li ${ }^{1,2}$, Wei Fu ${ }^{1,2}$, Ying Liu ${ }^{1}$, Shan-Shan Geng ${ }^{1}$, Jiu-Ye Guo ${ }^{1}$, Yue-Qiao \\ Gao ${ }^{1}$ and Hong-Lei $\mathrm{Li}^{1}$
}

${ }^{1}$ Department of Neonatology and NICU, Beijing Chaoyang District Maternal and Child Health Care Hospital, Beijing 100101, China

${ }^{2}$ Department of Neonatology and NICU of Bayi Children's Hospital, the Army General Hospital of the Chinese PLA, Beijing 100700, China

"Corresponding author: Department of Neonatology and NICU, Beijing Chaoyang District Maternal and Child Health Care Hospital, Beijing 100101, China. Email: liujingbj@live.cn

Received 2017 November 23; Revised 2018 March 05; Accepted 2018 April 07.

\begin{abstract}
Background: Neonatal stroke (NS) is a common, severe central nervous system disease that is the main cause of death and disability in neonates. Therefore, great attention has already been paid to it by clinicians. However, much knowledge remains yet to be discovered about this disease.

Objectives: To understand the clinical features, increase awareness, reduce missed diagnoses or misdiagnoses, and improve the prognosis of NS.

Methods: A retrospective analysis was conducted on clinical data from 51 patients with NS recording perinatal factors, clinical manifestations, imaging findings, and treatment outcomes.

Results: 1) Logistic multi-factor regression analysis showed that cesarean section, perinatal hypoxia, small for gestational age and maternal-fetal infection were the main risk factors of NS, 2) A total of $84.3 \%$ of the patients presented neurological symptoms or signs, of which $78.4 \%$ had convulsions as the primary symptom or major manifestation, and $67.5 \%$ had positioning significance, while $15.7 \%$ of the patients had no clinical manifestations, 3) Onset of symptoms: $76.7 \%$ within 24 hours after birth, $86.0 \%$ within 48 hours, $90.7 \%$ within 72 hours, and 7.0\% after 3 days, with only $2.0 \%$ after 7 days, 4 ) Bilateral hemisphere involvement was present in $49.1 \%$ of cases, left hemisphere involvement in 31.4\%, right hemisphere and basal ganglia involvement in $19.6 \%$ each, 5) The incidence of sequelae in living babies was $45.8 \%$, and the overall rate of a poor prognosis was $49.1 \%$.

Conclusions: 1) Convulsion was the most common primary symptom of NS, 15.7\% of the patients had no clinical manifestations. 2) The patients had an NS onset within several minutes to hours after birth, with $>75 \%$ having an onset within 24 hours, $85 \%$ within 48 hours, and $>90 \%$ within 72 hours after birth, 3) Caesarean section, perinatal hypoxia and small for gestational age were closely associated with NS, 4) Patients often exhibited bilateral hemisphere injury.
\end{abstract}

Keywords: Stroke, Cesarean Section, Convulsion, Newborn Infants

\section{Background}

Neonatal stroke (NS) also known as neonatal cerebral infarction, perinatal cerebral infarction, or perinatal stroke, is an area of damaged cerebral tissue resulting from either disruption of the blood flow in a major cerebral artery due to thrombosis or embolism (perinatal arterial ischemic stroke) or thrombosis in a major cerebral vein (cerebral sinus venous thrombosis), which occurs between 20 weeks of fetal life and 28th postnatal day and can be confirmed by neuroimaging or neuropathologic studies (1, 2 ). Recently, a growing number of patients has been diagnosed with NS, which has emerged as the second most common cause of neonatal convulsions and has placed a heavy burden on individuals and families (3). For further understanding of NS and to reduce the incidence rates of missed diagnosis, misdiagnosis, and mistreatment, we summarized the clinical features of NS in 51 patients admitted and treated in recent years.

\section{Methods}

\subsection{Clinical Information Collection}

The ethics committee and institutional review board of Beijing Chaoyang district maternal and child healthcare hospital approved this study. From January 2012 to June, 2017, a total of 51 patients were included in this study. The 
related information was recorded including general demographic information, mode of delivery, severe fetal distress and birth asphyxia, gestational ages (GA); premature rupture of membrane (PROM), congenital cerebrovascular malformations (CCM), small for gestational age (SGA), gender, birth weight, hematocrit, management methods and outcomes were collected in this study. The diagnosis of NS was based on the case history, clinical manifestations, the brain ultrasound and/or magnetic resonance findings.

The data analysis was conducted using SPSS 18.0 for Windows. Significance levels between differences were assessed using $\chi^{2}$ tests and logistic regression analysis. $\mathrm{P}<$ 0.05 was considered to be statistically significant.

\section{Results}

\subsection{General Demographic Information and Possible Reasons}

Listed in Table 1 logistic multi-factor regression analysis showed that the main risk factors of NS included caesarean section (odds ratio [OR]: 9.23; 95\% confidence interval $[\mathrm{CI}]: 5.39$ - 15.19), peri-natal hypoxia (fetal distress and birth asphyxia) (OR: 7.183; 95\% CI:3.29-15.43), SGA(OR: 6.99; 95\% CI:3.00 - 9.11), maternal-fetal infection (OR:3.38; 95\% CI: 2.87 - 5.89) were the main risk factors of NS.

\subsection{Main Clinical Manifestations}

As outlined in Table 2, 84.3\% of the patients presented neurological symptoms, and $78.4 \%$ had convulsions as the primary symptom or the major clinical manifestation. Among those patients with convulsion, 27 (67.5\%) patients had positioning significance. The symptoms included individual and unilateral limb twitching, mouth twitching, fixed gaze, systemic convulsions, or apnea, among others. The inhibited states manifested as weak response, poor mental health, poor sucking response, lethargy, and hypotonia, among others. However, 8 (15.7\%) patients had no clinical symptoms and the diagnosis was based on brain imaging findings. Four patients manifested with a significant puffy red spotted rash over the whole body, especially on the face and all limbs; these individuals were diagnosed as having incontinent a pigment, which was confirmed by genetic testing.

As shown in Table 3, the symptom manifested itself in more than $75 \%$ within 24 , in $85 \%$ within $48 \mathrm{~h}$, and in $90 \%$ within $72 \mathrm{~h}$ after birth, only in $1(2.3 \%)$ case symptom onset occurred more than $7 \mathrm{~d}$ after birth.

\begin{tabular}{|c|c|}
\hline Category & No. (\%) \\
\hline \multicolumn{2}{|l|}{ Gender } \\
\hline Male & $28(54.9)$ \\
\hline Female & $23(45.1)$ \\
\hline GA & $29-41+1 W$ \\
\hline Term & $46(90.2)$ \\
\hline Preterm & $5(8.8)$ \\
\hline \multicolumn{2}{|l|}{ Delivery mode } \\
\hline Vaginal & $14(27.5)$ \\
\hline Cesarean & $37(72.5)$ \\
\hline Birth weight, $g$ & 1050 - 4100 (one case unknown) \\
\hline \multicolumn{2}{|l|}{ Possible risk factors } \\
\hline Perinatal hypoxia & $26(51)$ \\
\hline Severe birth asphyxia & $4(7.8)$ \\
\hline PROM & $8(15.7)$ \\
\hline Purulent meningitis & $4(7.8)$ \\
\hline Erythrocytosis & $7(13.7)$ \\
\hline Incontinent a pigment & $4(7.8)$ \\
\hline CCM & $2(3.9)$ \\
\hline SGA & $31(60.8)$ \\
\hline \multicolumn{2}{|c|}{$\begin{array}{l}\text { Abbreviations: CCM, congenital cerebrovascular malformation; GA, gesta- } \\
\text { tional age; SGA, small for gestational age; PROM, premature rupture of mem- } \\
\text { brane. }\end{array}$} \\
\hline \multicolumn{2}{|c|}{ Table 2. Major Neurological Symptoms in this Group } \\
\hline Neurological Symptoms & No. $(\%)$ \\
\hline Convulsions & $43(84.3)$ \\
\hline Excited and irritated state & $6(11.8)$ \\
\hline Inhibited state & $15(29.4)$ \\
\hline Attenuation of the primary reflex & $14(27.4)$ \\
\hline No clinical findings & $8(15.7)$ \\
\hline \multicolumn{2}{|c|}{ Table 3. Onset Time of the Symptoms After Birth } \\
\hline Onset Time & No. $(\%)$ \\
\hline Within 24 hours & $33(76.7)$ \\
\hline Within 48 hours & $37(86.0)$ \\
\hline Within 72 hours & $39(90.7)$ \\
\hline Within 1 week & $50(98.0)$ \\
\hline
\end{tabular}

\subsection{Imaging Examinations}

All patients were diagnosed by imaging examinations, including brain ultrasound, CT scan, and magnetic resonance imaging (MRI). The brain ultrasound was used first 
as a screening method, after which, brain CT (some of the earliest cases) or MRI (recent years) was used to confirm the diagnosis. Congenital cerebrovascular malformations were confirmed by magnetic resonance angiography.

\subsection{Treatments}

The following treatment measures were used:

- Intravenous injection of phenobarbital sodium was preferentially used for patients with convulsions at a loading dose of 15 - $20 \mathrm{mg} / \mathrm{kg}$ and maintenance dose of 5 $\mathrm{mg} / \mathrm{kg} / \mathrm{d}$ twice daily at 12-hour intervals.

- Intravenous infusion of the compound Dan Shen Agent was used to clear the micro-circulation and to improve cerebral blood perfusion: at each time point, $2 \mathrm{~mL} / \mathrm{kg}$ of drug was added to $20 \mathrm{~mL}$ of $5 \%$ glucose solution and administered once daily for 10 - 14 days per course of treatment.

- Intravenous infusion of monosialotetrahexosylganglioside or 1,6- fructose diphosphate was used to protect or facilitate the recovery of injured brain cells: 10 - 14 days per course of treatment.

- Aspirin anticoagulant therapy was used for 3 patients at the dose of $50 \mathrm{mg} / \mathrm{kg} / \mathrm{d}$ three times orally with no significant adverse reactions.

- Hyperbaric oxygen: After a definite diagnosis and the parents' agreement, the patients were treated with conventional hyperbaric oxygen therapy once daily for 1 hour per session for 7-10 days per course of treatment. Two to three courses were used continuously according to the disease condition.

- Symptomatic supportive therapy: Normal blood pressure and fluid, electrolytes, and blood sugar stability were maintained, and the primary disease was actively treated.

- Others: The majority of NS cases had no significant elevation of intracranial pressure. Patients with a significant elevation of intracranial pressure were given an intravenous infusion of mannitol ( $0.25-0.5 \mathrm{~g} / \mathrm{kg})$ for $6-8$ hours each time according to the disease condition, and the dose was reduced gradually.

- Rehabilitation training was given as soon as the disease condition was stable.

\subsection{Prognosis}

Two of the 51 patients died, accounting for a case fatality rate of $3.9 \%$. Forty-nine patients survived to be discharged and were followed up from 6 months to 6 years, 1 of them died, and 22 cases had various sequelae. The incidence of sequelae in survived babies was $45.8 \%$, including hydrocephalus, cerebral palsy, hemiplegia, dyskinesia, epilepsy, and cognitive disorder. The overall rate of a poor prognosis (including death and sequelae) was $49.0 \%$ (25/51) in this group.

\section{Discussion}

\subsection{Stroke Is a Common Neonatal Disease}

The incidence of NS is closely associated with birth weight, with a lower birth weight leading to a higher incidence. Several studies have reported neonatal hemorrhagic stroke. Bassan et al. (4) reported the incidence rates of hemorrhagic cerebral infarction in low-birth-weight infants to be $10 \%$ with a birth weight $<750 \mathrm{~g}, 2.2 \%$ with a birth weight of $750-1500 \mathrm{~g}, 0.1 \%$ with a birth weight of 1500 $2500 \mathrm{~g}$, and 1/2300 - 4000 in full-term infants (5). According to the epidemiological survey data of Armstrong-Wells et al. (6), the incidence of neonatal hemorrhagic cerebral infarction is 6.2//100.000 total live births.

\subsection{Clinical Characteristics of NS}

\subsubsection{Clinical Manifestations}

The 51 NS patients had the following features:

- Convulsion was the most common clinical manifestation and was found in almost $80 \%$ of the patients; it was the primary symptom, and $2 / 3$ of our patients had localized significance. The cases included individual and unilateral limb twitching, mouth twitching, fixed gaze, systemic convulsions, or apnea. Thus, the possibility of stroke should be considered for patients with convulsions as the major clinical manifestation, particularly in those with positioning signs (2).

- Inhibited state: one-quarter of the patients presented an inhibited state as the major clinical manifestation, such as a weak response, poor mental health, poor sucking re-sponse, lethargy, low muscle tone, and an attenuated primitive reflex. The above manifestations could occur singly or in combination and were found in $47.1 \%$ of the patients. 
- NS could have no clinical manifestations: $15.7 \%$ of the patients had no clinical manifestations, with infarcts found only in the brain MRI. Therefore, the awareness and un-distending of NS need to be strengthened, and imaging examinations should be per-formed in a timely manner to help confirm the diagnosis (Figure 1).

- Early onset: The patients experienced an attack within several minutes to hours after birth. Specifically $>75 \%$ of the patients had an attack within 24 hours after birth, $>85 \%$ within 48 hours, and $>90 \%$ within 72 hours. The earliest onset occurred 10 minutes, and only 1 patent had an attack more than 1 week (12 days) after birth.

- Bilateral cerebral hemisphere involvement was found in nearly $50 \%$ of the patients. Left cerebral hemisphere involvement accounted for $1 / 3$ of the cases, and right cerebral hemisphere involvement accounted for nearly $1 / 5$ of the cases. This result differed from a previous report in the literature that stated that left hemisphere involvement was most common (2). The parietal lobe was most commonly involved, followed by the frontal lobe, the temporal lobe, and the occipital lobe; these lobes were individually or simultaneously involved. Basal ganglia infarction was also common; it was present in eight (15.7\%) patients in the present study. Basal ganglia infarction could also exist alone. Husson et al. (7) reported that infarction in the middle cerebral artery distribution area was most common in neonatal cerebral arterial ischemic stroke and was found in up to $73 \%$ of cases.

\subsubsection{Causes and High-Risk Factors}

Logistic multi-factor regression analysis showed that cesarean section, perinatal hypoxia, SGA and maternalfetal infection were the main risk factors of NS. Other risk factors included erythrocytosis, cerebrovascular malformations, and genetic disease, among others (1). Cesarean section: Our results showed that caesarean section was closely associated with NS. Cesarean delivery accounted for $>70 \%(72.5 \%)$ of our patients, $90 \%$ of which were delivered by emergency cesarean section. This finding suggests that cesarean delivery, particularly emergency cesarean section, may be an important high-risk factor for NS, which warrants increased attention from clinicians.

The underlying reason for this relationship remains unclear but may be related to the fact that patients who require cesarean delivery are often associated with a certain perinatal risk factor that affects automatic cerebrovascular regulation and initiates the endogenous coagulation mechanism. One study reported that emergency cesarean sections had a certain relationship with neonatal hemorrhagic stroke (6). However, the number of cases is still small, and the exact relationship between cesarean section and NS needs further investigation. 2) Perinatal hypoxia: This condition is a common cause leading to NS. In the present study, $>50 \%$ of the patients had a history of perinatal hypoxia, mainly fetal bradycardia and fetal distress ( $~ 85 \%)$, whereas severe birth asphyxia accounted for only a small portion of the patients $(\sim 15 \%)$. A largesample epidemic-logical survey in Switzerland found that intrauterine fetal distress was an independent risk factor for NS (5). Therefore, monitoring of perinatal hypoxia must be strengthened, particularly in cases of intrauterine distress of the fetus.

The possible mechanisms underlying hypoxia leading to NS are as follows:

- Apnea hypoxia induces focal cerebrovascular occlusion.

- Hypoxia-induced energy metabolism disorder, acidosis, intracellular and extracellular water and ion balance disorder, abnormal calcium ion distribution, abnormal phospho-lipid metabolism, lipid peroxidation, free radicals, and excitatory amino acid neuro-toxicity are involved in the pathogenesis of NS.

- Hypoxia-induced inflammatory responses, inflammatory cells, cytokines, and platelet-activating factors are all important media of NS (3).

Premature rupture of fetal membranes (PROM), intrauterine infection, and purulent meningitis are important high-risk factors for NS. Twelve (23.5\%) patients were associated with perinatal infection or purulent meningitis. The latest international studies have also posited that perinatal infection (maternal fever and early onset sepsis) and purulent meningitis are important risk factors for ischemic stroke in full-term infants $(8,9)$, which warrants attention. The possible mechanisms are as follows: in severe infection, various pathogenic microorganisms and their metabolites and toxins activate the immune system, the cerebral vessels undergo allergic reactions leading to intimal thickening and occlusion of the cerebral arteries; infection-induced cerebral vasculitis, arachnoiditis, and ventriculitis result in secondary cerebral edema and cerebral softening, which can directly lead to cerebral infarction; bacterial endotoxins lead to cerebral blood flow reduction and a rise in temperature, which increase the susceptibility of immature brain tissues to injury and induce or aggravate brain damage (10). Erythrocytosis: is also a common risk factor for NS. Seven (13.7\%) cases of NS in this study were due to erythrocytosis. The significant 

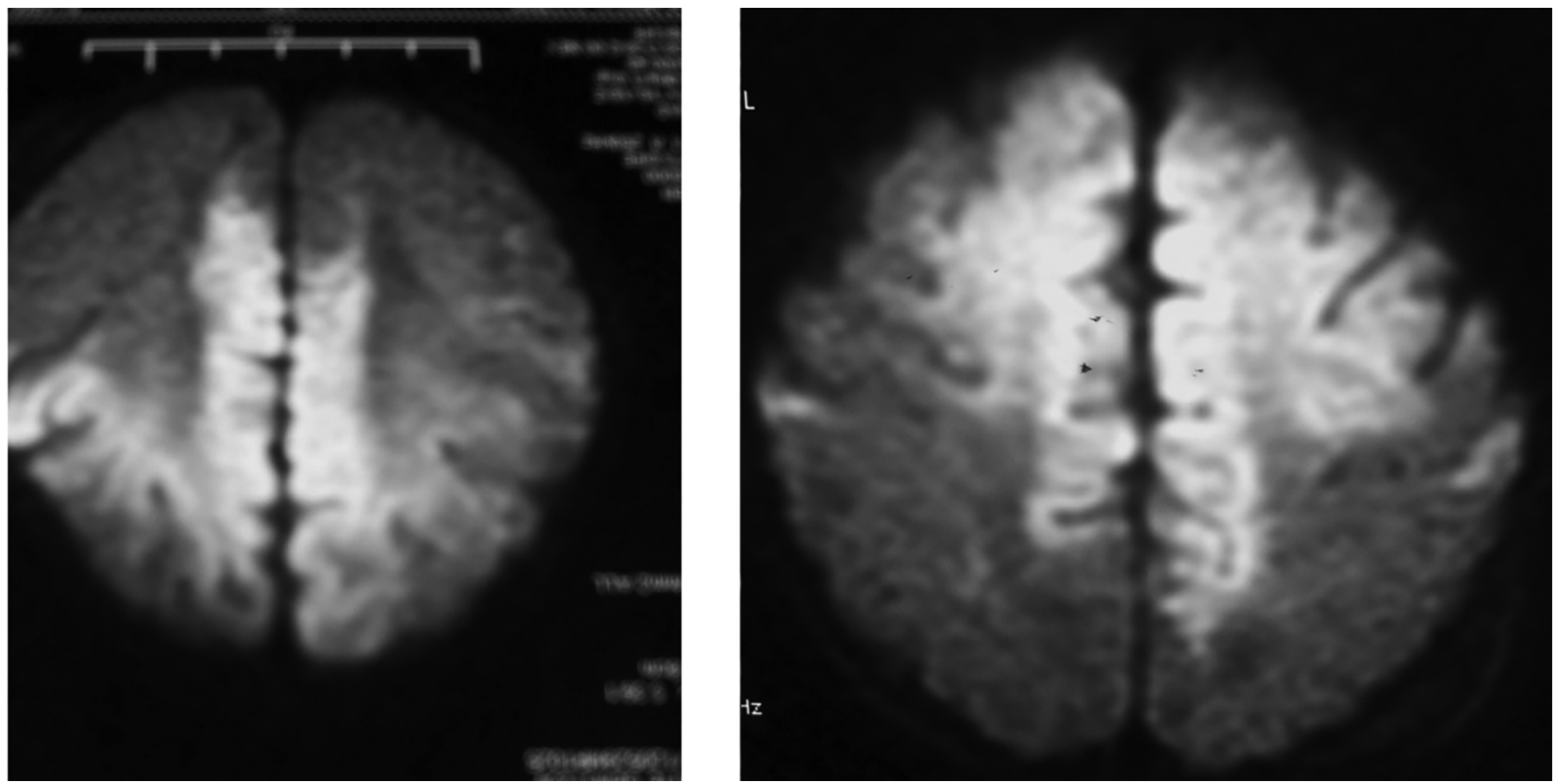

Figure 1. A patient who was asymptomatic and was not excluded from cerebral infarction G1P1, $38+3 \mathrm{w}$, vaginal delivery, birth weight $3200 \mathrm{~g}$, without intrauterine distress, Apgar 7, 9 and 10 points in 1,5 and 10 min, respectively. The patient had a good general condition after birth with primitive reflex induced normally and no clinical symptoms. However, a brain MRI showed large areas of infarct in the brain parenchyma on the bilateral occipital lobe and both sides of the brain midline. MRI re-examination 3 weeks later showed formation of a softening lesion at the originally infarcted site.

increase in red blood cells results in increased blood viscosity and slow blood flow, thereby inducing small blood vessel embolism and leading to is chemic necrosis of the brain tissue (11). SGA: The results of this study showed that SGA was closely corrected with NS, the reason remains unknown, but maybe corrected with most of these babies accompanied with erythrocytosis (4). Cerebrovascular malformations: The possibility of congenital cerebrovascular malformations should be considered for NS with an unknown etiology. Here, two patients were admitted to the hospital for unexplained convulsions without any perinatal risk factors or abnormalities in their laboratory examinations. Cerebral infarction was detected by a brain MRI. In addition, middle cerebral arterial malformations in the left and right hemispheres were confirmed by MRI angiography (5). Genetic disease: Four patients with incontinent a pigment had concomitant stroke. Two patients were admitted to the hospital due to a systemic rash after birth. Incontinent a pigment was confirmed by a skin biopsy and genetic testing. No patients had significant neurological symptoms or signs. Nonetheless, the presence of multiple, focal infarcts was confirmed by a brain MRI examination (Figure 2).

\subsubsection{Prognosis}

The overall rate of a poor prognosis was close to $60 \%$ in our patients, which was similar to the previously reported results (9). Based on the results of this study and previous reports, the chance of a poor prognosis increases in patients with the following conditions (12):

- Premature hemorrhagic cerebral infarction nearly $40 \%$ of the patients with this condition died, and $86 \%$ of the survivors developed spastic paraplegia or asymmetric tetraplegia and mental retardation.

- Patients with convulsions in the neonatal period and significant neurological abnormalities at discharge.

- The coexistence of cerebral hemisphere, internal capsule, and basal ganglia injuries confirmed by an MRI examination.

- Patients showing abnormal activity in the unilateral or bilateral background on the electroencephalogram. These patients were likely to have the sequelae of hemiplegia, and $>70 \%$ of the patients with a maximum activity $<10 \mu \mathrm{v}$ and/or minimum activity $<5 \mu \mathrm{v}$ on the $\alpha$ electroencephalogram had a poor prognosis.

- Patients with bilateral basal ganglia involvement: Rutherford et al. (2) reported that $94 \%$ of patients with 

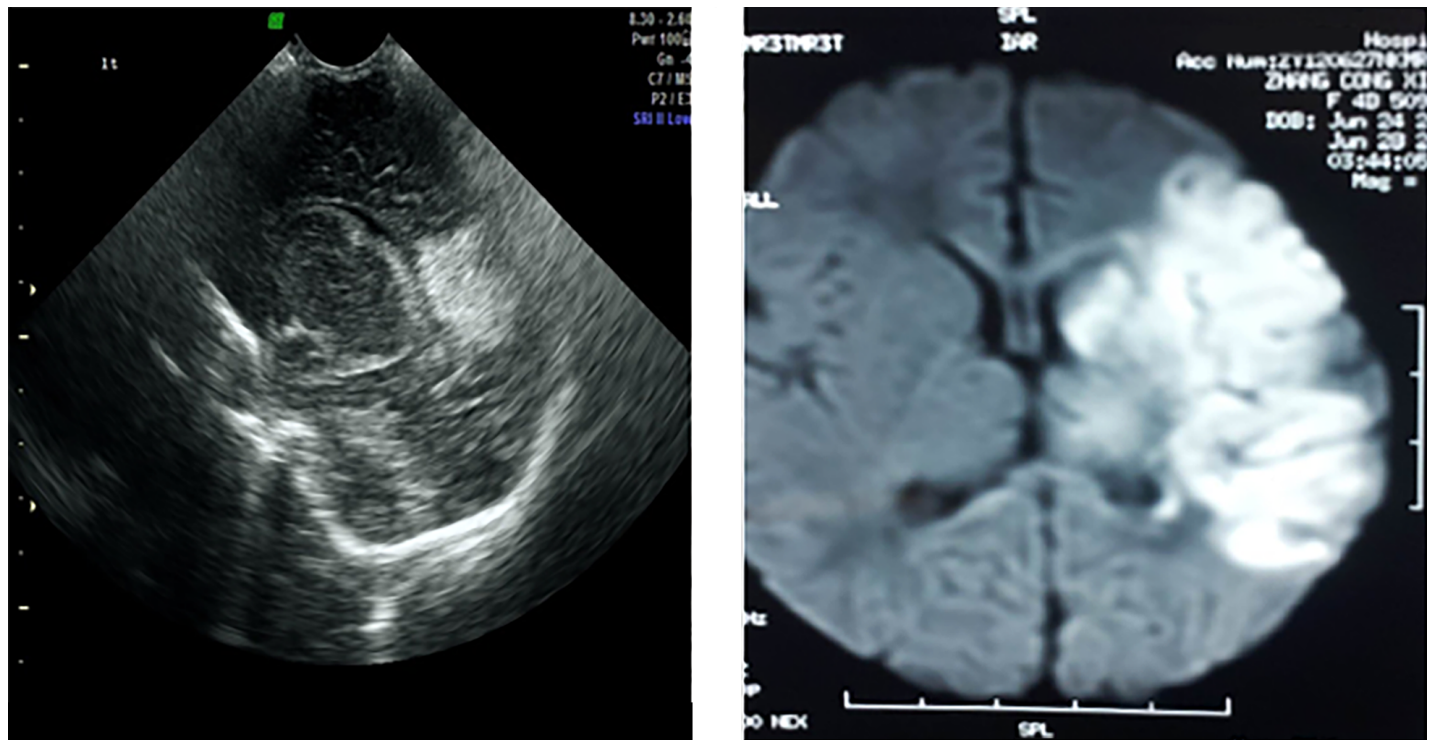

Figure 2. Gestational age $40+2 \mathrm{w}$, birth weight $3220 \mathrm{~g}$. Changed to cesarean delivery after failure of tried labor with no asphyxia at birth. Convulsions shortly after birth mainly in the right limbs. Brain ultra-sound at $48 \mathrm{~h}$ after birth showed a typical "wedge"-shaped echo enhanced area in the lateral upper side of the triangle zone of the left lateral ventricle. Brain MRI-DWI confirmed infarction in the distribution area of the middle arterial branches in this side of the brain.

cerebral ischemic stroke who had no involvement of the corticospinal tract, presented no movement disorders, whereas $66 \%$ of patients with involvement of the corticospinal tract had the hemiplegia sequel, and $88 \%$ of isolated subcortical infarction in the middle cerebral artery distribution area had no movement disorder sequelae.

\subsection{Diagnosis}

The diagnosis depends on imaging examinations. Both brain ultrasound and diffusion-weighted MRI (DWI) can provide an early diagnosis of NS (13). Brain ultra-sound is the first option and is sensitive to the diagnosis of infarction in the anterior and middle cerebral arterial blood supply regions (Figure 3). MRI is presently the most reliable means of diagnosing NS, whereas DWI is the most sensitive method capable of showing cerebral ischemic changes within minutes of onset (14). The infarcted area is manifested as a low signal on T1, a high signal on T2 in a conventional MRI, and a significant high signal in DWI. Cerebrovascular malformation is another important cause of NS. MRI angiography can be used for patients who are suspected of having or who are not excluded from having cerebrovascular malformations.

\subsection{Treatment}

There is no specific effective treatment for NS. Symptomatic and supportive treatment is mainly used in the acute phase with a principle of increasing the cerebral blood flow, preventing disease progression, and achieving etiological treatment. We combined the application of Dan Shen agents and hyperbaric oxygen therapy for NS treatment and obtained satisfactory clinical effects, although neither of the treatments were evidence-based, and "further high-quality randomized controlled trials about using Dan Shen agents for acute ischemic stroke should be performed" (15). Antithrombotic therapy was not recommended for neonatal cerebral infarction (16), but a more recent study offered the opposite opinion. Kirton et al. (17) reported that prompt diagnosis of neonatal cerebral sin venous thrombosis (CSVT) is essential because it is a treatable disorder with a natural history of early progression; thus, anticoagulation is increasingly accepted as routine practice for neonatal CSVT without hemorrhage. Jordan et al. (18) found that whether stroke patients progressed into epilepsy was mainly related to the volume of the cerebral infarction and that long-term anticonvulsion therapy in the neonatal period could not reduce the chance of progression into epilepsy. Rehabilitation treatment is mainly used in the recovery phase to continuously maintain brain functions and to promote brain rehabilitation. 

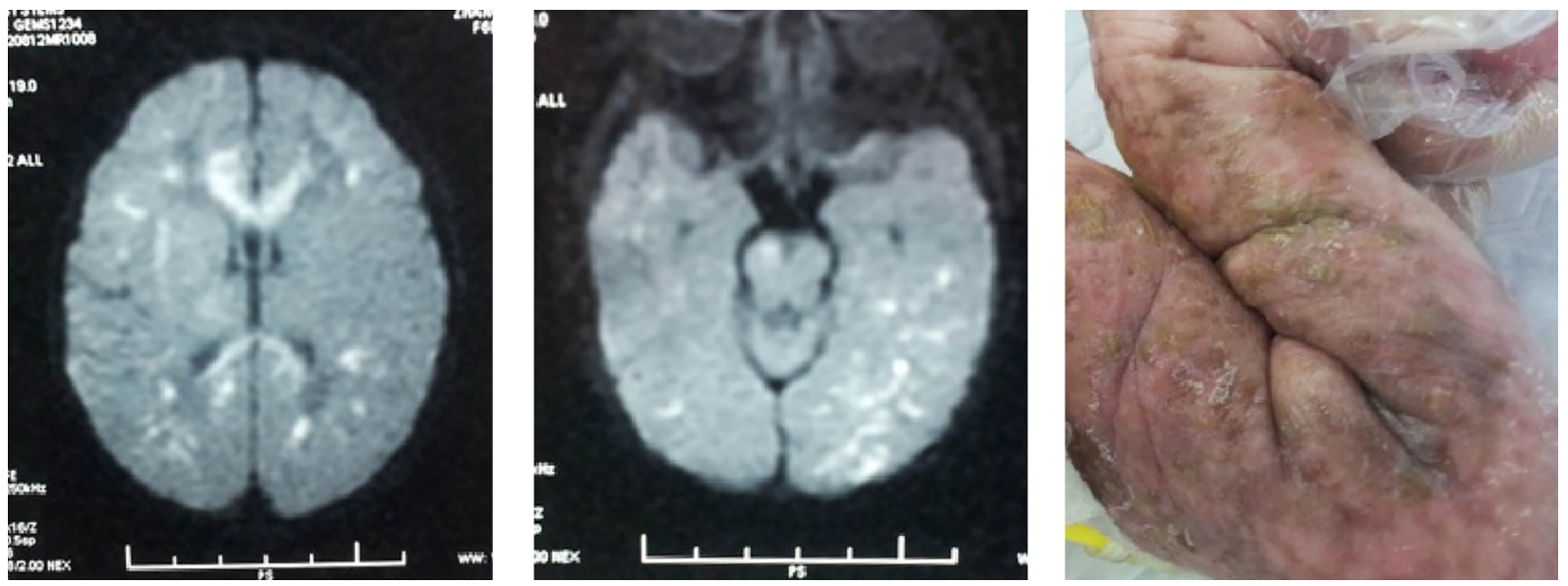

Figure 3. Case 3, Incontinent a pigment: G1P1, male, gestational age $38+2 \mathrm{w}$, cesarean delivery, birth weight $3160 \mathrm{~g}$. The brain MRI showed a diffuse focal infarct in the bilateral cerebral hemispheres that was particularly significant in the left parietal lobe.

\section{Footnote}

Authors' Contribution: Jing Liu contributed to the study conception, data collection and analysis, write and approval of the manuscript. Ru-Xin Qiu contributed to clinical data collection and analysis, manuscript preparation, and approval of the final manuscript. Jian-Jun Li contributed to clinical data analysis, manuscript preparation, and approval of the final manuscript. Ying Liu contributed to clinical data collection, manuscript revision, and approval of the final manuscript. Wei Fu contributed to data collection, manuscript revision, and approval of the final manu-script. Every authorc equally to this study.

\section{References}

1. Raju TNK, Nelson KB, Ferriero D, Lynch JK. Ischemic perinatal stroke: summary of a workshop sponsored by the national institute of child health and human development and the national institute of neurological disorders and stroke. Pediatr. 2007;120(3):609-16. doi: 10.1542/peds.2007-0336.

2. Rutherford MA, Ramenghi LA, Cowan FM. Neonatal stroke. Arch Dis Child Fetal Neonatal Ed. 2012;97(5):F377-84. doi: 10.1136/fetalneonatal2010-196451.

3. Lynch JK, Hirtz DG, DeVeber G, Nelson KB. Report of the National Institute of Neurological Disorders and Stroke workshop on perinatal and childhood stroke. Pediatrics. 2002;109(1):116-23. [PubMed: 11773550].

4. Bassan H, Feldman HA, Limperopoulos C, Benson CB, Ringer SA, Veracruz E, et al. Periventricular hemorrhagic infarction: risk factors and neonatal outcome. Pediatr Neurol. 2006;35(2):85-92. doi: 10.1016/j.pediatrneurol.2006.03.005.

5. Schulzke S, Weber P, Luetschg J, Fahnenstich H. Incidence and diagnosis of unilat-eral arterial cerebral infarction in newborn infants. $J$ Perinat Med. 2005;33(2):170-5. doi: 10.1515/jpm.

6. Armstrong-Wells J, Johnston SC, Wu YW, Sidney S, Fullerton HJ. Prevalence and predictors of perinatal hemorrhagic stroke: results from the kaiser pediatric stroke study. Pediatrics. 2009;123(3):823-8. doi: 10.1542/peds.2008-0874. [PubMed: 19255009].

7. Husson B, Hertz-Pannier L, Renaud C, Allard D, Presles E, Landrieu P, et al. Motor outcomes after neonatal arterial ischemic stroke related to early MRI data in a prospective study. Pediatrics. 2010;126(4):912-8. doi: 10.1542/peds.2009-3611. [PubMed: 20855393].

8. Harteman JC, Groenendaal F, Kwee A, Welsing PMJ, Benders MJNL, de Vries LS. Risk factors for perinatal arterial ischaemic stroke in fullterm infants: a case-control study. Arch Diseas Child Fetal Neonatal Ed. 2012;97(6):F411-6. doi: 10.1136/archdischild-2011-300973.

9. Hernandez MI, Sandoval CC, Tapia JL, Mesa T, Escobar R, Huete I, et al. Stroke patterns in neonatal group B streptococcal meningitis. Pediatr Neurol. 2011;44(4):282-8. doi:10.1016/j.pediatrneurol.2010.11.002.

10. Gardner MA, Hills NK, Sidney S, Johnston SC, Fullerton HJ. The 5-year direct medical cost of neonatal and childhood stroke in a population-based cohort. Neurol. 2010;74(5):372-8. doi: 10.1212/WNL.ob013e3181cbcd48.

11. Kirton A, Armstrong-Wells J, Chang T, deVeber G, Rivkin MJ, Hernan$\operatorname{dez} \mathrm{M}$, et al. Symptomatic neonatal arterial ischemic stroke: the international pediatric stroke study. Pediatr. 2011;128(6):e1402-10. doi: 10.1542/peds.2011-1148.

12. Roze E, Kerstjens JM, Maathuis CGB, ter Horst HJ, Bos AF. Risk factors for adverse outcome in preterm infants with periventricular hemorrhagic infarction. Pediatr. 2008;122(1):e46-52. doi: 10.1542/peds.20073305.

13. Roze E, Van Braeckel KNJA, van der Veere CN, Maathuis CGB, Martijn A, Bos AF. Functional outcome at school age of preterm infants with periventricular hemorrhagic infarction. Pediatr. 2009;123(6):1493500. doi: 10.1542/peds.2008-1919.

14. Cowan F. Does cranial ultrasound imaging identify arterial cerebral infarction in term neonates? Arch Dis Child Fetal Neonat Ed. 2005;90(3):F252-6. doi:10.1136/adc.2004.055558.

15. Wu B, Liu M, Zhang S. Dan Shen agents for acute ischaemic stroke. Cochrane Da-tabase Syst Rev. 2007;18(2). CD004295. doi: 10.1002/14651858.cd004295.

16. Wusthoff CJ, Kessler SK, Vossough A, Ichord R, Zelonis S, Halperin A, et al. Risk of later seizure after perinatal ar-terial ischemic stroke: A prospective cohort study. Pediatr. 2011;127(6):e1550-7. doi: 10.1542/peds.2010-1577. 
17. Kirton A, deVeber G. Paediatric stroke: pressing issues and promising directions. Lancet Neurol. 2015;14(1):92-102. doi: 10.1016/s14744422(14)70227-3.

18. Jordan LC, Rafay MF, Smith SE, Askalan R, Zamel KM, deVeber G, et al.
Antithrombotic treatment in neonatal cere-bral sinovenous thrombosis: results of the international pediatric stroke study. J Pediatr. 2010;156(5):704-1000. doi: 10.1016/j.jpeds.2009.11.061. 